\title{
Artelogie
}

Recherche sur les arts, le patrimoine et la littérature de l'Amérique latine

5 | 2013

Femmes créatrices en Amérique latine : le défi de synthétiser sans singulariser

\section{Du salon à la scène : l'ascension des femmes et du piano au Brésil du vingtième siècle}

Dalila Vasconcellos de Carvalho

\section{OpenEdition}

Journals

Édition électronique

URL : https://journals.openedition.org/artelogie/5417

DOI : 10.4000/artelogie.5417

ISSN : 2115-6395

Éditeur

Association ESCAL

Référence électronique

Dalila Vasconcellos de Carvalho, « Du salon à la scène : I'ascension des femmes et du piano au Brési du vingtième siècle », Artelogie [En ligne], 5 | 2013, mis en ligne le 16 octobre 2013, consulté le 20 décembre 2021. URL : http://journals.openedition.org/artelogie/5417 ; DOI : https://doi.org/10.4000/ artelogie. 5417

Ce document a été généré automatiquement le 20 décembre 2021.

Association ESCAL 


\title{
Du salon à la scène : l'ascension des femmes et du piano au Brésil du vingtième siècle
}

\author{
Dalila Vasconcellos de Carvalho
}

\section{NOTE DE L'AUTEUR}

Il est important de souligner que les œuvres qui ont été utilisées ici en langue portugaise n'ont pas été encore officiellement traduites en langue française. Donc, les traductions libres sont de ma responsabilité.

1 Cette plainte fut exprimée par le compositeur et pianiste Ernesto Nazareth (1863-1934) en 1930, au Teatro Municipal de Rio de Janeiro pendant un concert de la pianiste Guiomar Novaes (1894-1979)2 ${ }^{2}$, qui avait reçu le premier prix du Conservatoire de Paris $^{3}$ en 1911, à l'âge de 17 ans. Celle-ci a développé un riche parcours de concertiste, tout comme deux autres pianistes brésiliennes: Antonietta Rudge (1885-1974) et Magda Tagliaferro (1893-1986). Ces trois femmes furent les premières pianistes du Brésil à réussir à se bâtir une carrière d'envergure internationale en tant que concertistes au début du vingtième siècle.

2 Pour quelles raisons Nazareth n'a-t-il pas réussi à devenir concertiste comme Guiomar, Magda et Antonietta? Pourquoi dit-il qu'il aurait voulu aller en Europe? Pour quelles raisons a-t-il échoué à s'y rendre?

3 Claquant la porte pendant le spectacle, Nazareth exprima alors une frustration qui l'a accompagné toute sa vie malgré la réputation qu'il avait acquise comme pianiste et compositeur durant la Belle Époque de la ville de Rio de Janeiro.

4 La période qui suivit la fondation de la République en 1889, se caractérise par la modernisation accélérée de Rio de Janeiro, dans un contexte politique de retour à la stabilité grâce au gouvernement de Campos Sales (1898-1902) et ses élites. C'est toutefois durant le mandat de Rodrigues Alves (1902-1906), avec l'arrivée au pouvoir de 
Pereira Passos (1863-1913) comme maire, que le processus de métamorphose de la capitale de la République, Rio de Janeiro, atteint son apogée, notamment sous l'effet des importantes transformations urbaines inspirées par Haussmann ${ }^{4}$. Du point de vue culturel, cette période est marquée par un "cosmopolitisme agressif » porté par une élite revendicative d'un progrès radical, transformant l'espace public, comme la vie et la pensée des « cariocas».

Ce processus, conflictuel il va sans dire, se manifestait par le rejet du passé colonial, et s'incarnait principalement dans le refus catégorique d'assumer l'héritage africain; il aura un impact important sur l'univers musical carioca. De ce fait, les frontières entre la «musique légère » et la «musique sérieuse » devinrent plus rigides, et elles furent progressivement appelées, l'une "musique populaire » et l'autre "musique érudite». La «musique populaire » fut jugée inférieure, dotée de défauts imparables, conduisant à mettre en doute tant la qualité de l'œuvre musicale que la réputation du musicien. Ainsi, certaines spécificités inhérentes aux musiques "populaires " commencèrent à être évitées par les musiciens. Les compositeurs voulant être reconnus comme « érudits » entreprirent de « masquer » certaines formes.

6 Lors de la période du Second Royaume, musiciens et compositeurs passaient régulièrement d'un milieu à l'autre sans que cela ne suscite de controverses : les pièces érudites apportaient un certain prestige, tandis que les compositions populaires permettaient de gagner en popularité et en appui. C'est ce que vécut le compositeur de l'opéra O Guarani, Carlos Gomes (1836-1896) .

7 La circulation des musiciens entre les deux milieux ne s'interrompit évidemment pas pendant la période républicaine, mais, désormais, les musiciens commencèrent à engager des stratégies visant à dissimuler leurs mouvements entre ces univers, par exemple en utilisant des pseudonymes ${ }^{7}$.

8 Dans le processus de modernisation de la ville, la vie sociale et culturelle connut une nouvelle dynamique, avec le développement du Théâtre de variétés ${ }^{8}$, des magasins de musique et du cinéma, qui étaient alors intimement liés (toutes les salles de cinéma étaient dotés d'un petit ensemble musical pour accompagner les films). Or, le piano était inséré dans ces trois milieux. Si à une époque antérieure, cet instrument était exclusivement réservé aux « érudits » et rattaché aux habitudes aristocratiques de la cour ${ }^{9}$, au début du vingtième siècle, il avait déjà gagné tous les espaces sociaux des villes de Rio de Janeiro et de Sao Paulo. On le trouvait dans les salles de concert et les théâtres, à l'Institut National de Musique et au Conservatoire Dramatique et Musical de Sao Paulo, mais aussi dans les lieux généralement fréquentés par la population des classes moyennes voire pauvres: au cinéma, dans les pâtisseries, les magasins de musique ou encore dans les espaces domestiques: lors de fêtes, il était commun aux gens de se réunir autour du piano et du " pianeiro $»^{10}$.

9 Face à un public avide de divertissements, un marché culturel prit un essor autour de trois éléments fondamentaux : le théâtre, le piano et les éditions de musique. Les pièces à succès au théâtre étaient immédiatement imprimées sur des partitions, vendues aux clients des magasins de musique où les pianistes les présentaient. La production musicale originaire de cette triade fut cependant jugée, de façon péjorative, comme étant à la fois " populaire ", « banale », " grossière » et sans valeur artistique ${ }^{11}$.

10 Dans un environnement où la présence d'une musique "populaire " était indéniable, par rapport aux stratégies cosmopolites d'une élite engagée dans des pratiques et des discours ségrégationnistes, les frontières entre les deux univers furent exacerbées par 
l'établissement de principes de classification des musiques sur la base de modèles européens. Durant le Second Royaume, la Cour établissait et hiérarchisait la valeur artistique; elle favorisait les études en Europe en attribuant des bourses d'études, en instituant des prix et en nommant les professeurs au Conservatoire Impérial de la Musique. Pendant la période républicaine, ce Conservatoire Impérial prit le nom d'Institut National de Musique, il consolida son assise et l'élan de la modernité par l'assimilation "des idées républicaines ", intégrant la « civilisation par la musique $»^{12}$. Il occupa une position centrale dans la définition de la hiérarchisation de la production musicale, selon les critères issus de la « musique occidentale $»^{13}$ et devint le bastion de la « musique érudite ». C'est dans ce contexte que la carrière d'Ernesto Nazareth offre un exemple représentatif de la métamorphose de l'univers musical. En effet, la carrière de ce musicien se déroule dans cette transition de l'Empire à la République, soit entre la popularisation du piano et l'émergence du récital. Une compréhension de sa frustration personnelle permettra en outre de témoigner de l'ascension des femmes dans l'univers pianistique au Brésil.

11 Nazareth naît dans une famille modeste en 1863. Son père, Vasco, était commissionnaire aux douanes du port de la ville de Rio de Janeiro et sa mère, Carolina, était probablement femme au foyer et pianiste amatrice. Ainsi, ce fut elle qui initia ses quatre enfants à la musique. Seul Nazareth continua ses études de piano. À l'âge de dix ans, suite au décès de sa mère, son père lui fit clairement savoir qu'il ne l'encouragerait pas dans une éventuelle carrière musicale et tenta d'interrompre ses études de piano. Cependant, Nazareth décida de les poursuivre avec un professeur privé, ce qui allait rapidement devenir coûteux pour une famille aussi pauvre que la sienne. Peut-être son père eut-il préféré qu'il accède à une profession mieux établie, estimant qu'une carrière de musicien ne saurait contribuer suffisamment aux besoins du foyer familial.

12 L'enseignement particulier du piano érudit continuait d'être assuré par des professeurs privés dont la plupart étaient des étrangers. Bien que les principales institutions musicales d'enseignement «érudit » et professionnel avaient déjà été créées par Dom Pedro II, celles-ci avaient pour obligation d'assurer le développement de l'opéra, qu'affectionnait particulièrement la Cour portugaise.

Le Conservatoire Impérial de Musique de Rio de Janeiro, fondé par Francisco Manuel da Silva (1795-1865) en 1848, est d'ailleurs un héritage de cette période. Le Conservatoire était rapidement devenu la principale institution de formation des musiciens de tout le pays. Son but était clairement affiché : offrir un enseignement " érudit » pour répondre aux exigences du théâtre lyrique et de l'opéra. Les enseignements étaient destinés à former des compositeurs, solistes, choristes, et instrumentistes (essentiellement instruments à cordes et à vent).

14 Pendant longtemps, l'enseignement du piano fut plus aléatoire. On lui octroya une place importante à partir de 1883 , avec la nomination du premier professeur, le pianiste et compositeur Carlos Cavalier Darbilly (1846-1918). ${ }^{14}$

15 Nazareth, à l'âge de 14 ans et de manière autodidacte, composa sa première pièce musicale, la polka Você bem sabe (1877), ironiquement ou malgré tout dédiée à son père. Cette pièce fut ensuite présentée à Arthur Napoleão, éditeur de musique ${ }^{15}$, qui entreprit de la publier ${ }^{16}$.

16 Le succès de cette publication le fit connaitre. Ses oncles, enthousiasmés par sa réputation, étudièrent la possibilité de lui permettre de prolonger son cursus en 
Europe, son plus grand rêve. Malheureusement, faute de ressources financières suffisantes, le projet ne se concrétisa pas.

Nazareth savait que pour devenir concertiste, il fallait étudier et obtenir un prix au sein d'un conservatoire européen. Peu de musiciens brésiliens avaient alors eu l'opportunité de compléter leur formation musicale en Europe. Pour ceux qui ne disposaient pas des moyens suffisants pour un tel voyage, la seule alternative était de demander une bourse d'études à Dom Pedro II ou de faire partie du cercle aristocratique, comme Luiza Leonardo (1859-1926). Ainsi, Dom Pedro II contribua-t-il directement à la carrière de plusieurs artistes (poètes, peintres et musiciens) en leur attribuant, selon sa volonté, une aide financière ou une bourse d'études pour parfaire leur art en Europe ${ }^{17}$.

Parmi ses boursiers, deux seulement furent des femmes. L'une d'entre elles, la pianiste Luiza Leonardo, se distingua grâce aux prix qu'elle reçut et à la carrière internationale qu'elle se construisit.

Luiza Leonardo ${ }^{18}$, née à Rio de Janeiro, en 1859, était la fille du portugais Victorino José Leonardo, professeur de musique à l'Institut Impérial des Garçons Aveugles ${ }^{19}$. Sa mère, Carolina de Oliveira Leonardo, descendante des Nassau, naquit dans l'État du Pernambouc. Ses origines nobles assuraient à la famille de Luiza un statut social élevé. Baptisée à l'église de Saint-Joseph, Luiza eut pour parrain Dom Pedro II lui-même. Son père étant professeur de musique, Luiza put commencer ses études de piano dès l'âge de sept ans. Plus tard, elle étudia auprès d'Isidoro Bevilacqua, pianiste et professeur de la Princesse Isabelle (1846-1921).

20 Comme le prescrivaient les règles de la société de l'époque en matière d'éducation pour les jeunes filles, la première présentation publique de Luiza ne se fit pas dans un espace domestique. Isidoro Bevilacqua nourrissait un plan plus ambitieux pour son élève, à savoir la présenter comme une enfant prodige. Aussi organisa-t-il un concert public au Théâtre Lyrique Provisoire, auquel assistèrent nombre de personnes illustres, parmi lesquelles les membres de la Famille royale. À la fin de la présentation, Luiza, alors âgée d'environ neuf ans, reçut de Dom Pedro II une bourse en vue de lui permettre d'aller étudier en Europe. Luiza fut sans doute la plus jeune artiste ayant bénéficié d'un tel privilège.

21 En 1873, à l'âge de 14 ans, Luiza fit ses débuts de compositrice avec la Grande Marche Triomphale, pièce dédiée à Dom Pedro II et jouée par l'Orchestre Pasdeloup ${ }^{20}$. En 1878, elle termina ses études du piano avec l'obtention du premier prix du Conservatoire de Paris, ce qui lui permit de prolonger son séjour en Europe.

Après avoir passé deux ans comme pianiste à la Cour de Louis I au Portugal, Luiza revint au Brésil vers 1880. Ce choix lui fut désastreux car elle se trouva dès lors dans l'impossibilité de poursuivre une carrière artistique: les commandes de concerts de piano solo étaient trop insuffisantes à Rio de Janeiro, et, en tant que femme, elle ne pouvait pas gagner sa vie dans cette voie. Ainsi dut-elle renoncer à cette perspective.

À la fin du dix-neuvième siècle, le piano était d'abord et avant tout un instrument réservé au " salon ${ }^{21}$. Sa pratique et son répertoire étaient essentiellement destinés à la vie sociale : aux bals, fêtes et réceptions. Le piano était perçu comme un instrument d'accompagnement. En Europe, compte-tenu du long processus social, culturel et artistique, des instrumentistes se produisaient déjà depuis longtemps comme solistes. Payés pour leurs prestations, certains pianistes virtuoses, comme Clara Schumann (1819-1896) et Franz Liszt (1822-1886), avaient alors un pouvoir d'attraction magistral, 
et furent considérés, elle, comme la première pianiste à entreprendre une carrière d'interprète et lui, le " père du récital moderne $»^{22}$.

$\mathrm{Au}$ Brésil, comme on l'a vu, les rares espaces existant pour la musique érudite étant occupés par l'opéra, il ne demeurait que les clubs musicau ${ }^{23}$ pour le récital de piano. Cette forme particulière de concert fut d'ailleurs introduite au Brésil par les pianistes étrangers. Dans la première moitié du dix-neuvième siècle, on comptait 37 pianistes étrangers à Sao Paulo et à Rio de Janeiro ${ }^{24}$. Parmi eux, se trouvaient quelques virtuoses, comme Thalberg (1812-1871), un pianiste considéré comme le grand rival de Liszt. Quelques-uns se produisirent en public dans les sociétés musicales.

Il est important de souligner qu'à cette époque, les femmes ne pouvaient pas fréquenter la vie culturelle, ni se faire admettre comme artistes dans la plupart des associations musicales, exception faite de la Princesse Isabelle qui avait l'habitude d'exposer ses talents musicaux dans le cadre de la Société des Concerts classiques, fondée en 1883 et dont elle était la patronne ${ }^{25}$.

26 Par contre, le Conservatoire Impérial de Musique de Rio de Janeiro, dès sa création, ouvrit ses enseignements aux femmes pour autant que les classes de femmes fussent séparées de celles des hommes. C'est ce que révèle la nomination de Leonor Tolentino de Castro, première professeure ${ }^{26} \mathrm{du}$ conservatoire, pour la classe exclusive des étudiantes de solfège ${ }^{27}$. La pratique musicale ${ }^{28}$ était permise aux femmes, à condition qu'elles respectent les règles de la société de l'époque, qui leur interdisaient d'envisager une carrière de pianiste professionnelle ${ }^{29}$.

En analysant la trajectoire de Luiza, nous sommes portés à penser que sa carrière de pianiste n'aurait jamais été interrompue si elle était restée en Europe. En effet, Luiza présentait un profil de pianiste virtuose: poussée par sa famille (son père était professeur de musique), elle avait bénéficié d'un apprentissage précoce de la musique, d'une formation érudite rigoureuse et d'un prix décerné par le conservatoire. De plus, si l'accès à une formation érudite du piano au Brésil était le privilège de jeunes filles de l'aristocratie; cette coutume se diffusa plus largement, les familles d'une classe moyenne émergeante voulant assurer une éducation identique à leurs filles.

28 Cependant, ces restrictions reliées aux questions de genre conduisaient les femmes à être, d'une part, presque confinées au piano dans leur éducation musicale, et d'autre part, assignées à le pratiquer en amatrices, sans pouvoir se présenter en salle de concert. Dès qu'elles étaient mariées, leurs obligations d'épouse et de mère devenaient prioritaires. En d'autres termes, limitée au salon et au dilettantisme, la pratique féminine du piano servait prioritairement à montrer et assoir la position sociale de la famille.

Réussissant à s'échapper de ce modèle rigide, la musicienne Chiquinha Gonzaga (1847-1935) $)^{30}$ constitua une exception parmi les femmes de son époque : elle accomplit sa carrière de pianiste et de compositrice au sein de l'univers masculin de la musique populaire. Chiquinha bénéficia d'une formation au piano, non pas pour satisfaire le désir personnel de ses parents de voir leur fille devenir une artiste : ni le père ni la mère n'avaient un lien avec la musique. En effet, quand son père José Basileu décida de donner une éducation musicale à sa fille, il voulait l'élever selon les modèles de l'époque, recommandant aux femmes des classes moyennes de la bourgeoisie, en cours d'émergence, de recevoir un enseignement musical, parmi d'autres activités telles que la cuisine, la broderie, la dentelle, l'apprentissage du français et la danse. 

intellectuelles et domestiques ne visait pas à former des musiciennes professionnelles. De plus, la pratique de l'instrument n'était nullement considérée comme une activité artistique. Elle faisait partie de l'éducation féminine, une activité un peu anodine en attendant le mariage. Même si le mariage ne mettait pas fin à la pratique du piano, celle-ci était destinée au divertissement. Le piano était situé dans le salon ou dans la salle de musique - deux espaces féminins de la maison, organisés de manière à faire monstration de la position sociale de la famille lors de réceptions. Le rôle de la femme consistait à les animer et les orner par des présentations musicales. Jouer du piano et/ ou chanter auprès du piano constituaient pour les femmes des façons de remplir cette fonction ${ }^{31}$.

31 Chiquinha continua ses leçons de piano jusqu'à environ 16 ans, moment où elle se maria avec Jacinto Ribeiro do Amaral, officier de la Marine Impériale. L'ironie du sort veut que le père lui offrit un piano pour constituer sa dot. Plus tard, ce piano deviendrait l'une des raisons de la séparation du couple. Après son mariage, Chiquinha ne relégua pas sa pratique du piano au second plan, derrière ses obligations d'épouse. Le mari, jaloux de l'attention que Chiquinha consacrait à une activité qui ne devait constituer qu'une occupation domestique secondaire, lui imposa de choisir entre luimême (le mariage) et le piano (son ambition artistique) : elle choisit le piano. Les conséquences en furent sévères : au moment de sa séparation, en 1869, Chiquinha fut reniée, d'une part par sa famille, qui lui interdisit de revoir ses enfants, et d'autre part par la société impériale.

fut son instrument de travail. Il subvint à ses besoins par ses concerts, la vente de ses compositions et les leçons qu'elle donnait. L'appui de Joaquim Antônio da Silva Callado (1848-1880), reconnu comme «le père du choro $»^{32}$, fut d'une importance déterminante pour sa carrière de pianiste et, plus tard, de compositrice. Callado la fit entrer dans l'univers masculin de la musique populaire, univers qui était alors tout à fait interdit aux femmes bénéficiant d'un statut social élevé.

Chiquinha s'y intégra " comme un homme » : elle commença à jouer du piano dans un groupe de choro de Callado à Rio de Janeiro et devint la première femme pianeira parmi les hommes pianeiros. Ensuite, elle joua dans les cafés-concerts, les pâtisseries, les bals, etc. Plus tard, elle entreprit de composer des pièces musicales pour la scène populaire qui l'avait accueillie. Par la suite, elle composera des pièces pour le Théâtre de variétés, intéressé par ce type de production populaire. Chiquinha s'établit de façon stable en tant que compositrice durant les deux dernières décennies du XIX ${ }^{\text {ème }}$ siècle, période où elle composa le plus et participa activement à la vie musicale de Rio de Janeiro.

Quand Nazareth débuta sa carrière, Chiquinha était déjà une compositrice populaire jouissant d'un réel succès. Elle poursuivait un chemin que Nazareth essaya toute sa vie d'éviter, renonçant tout autant à devenir un compositeur populaire qu'à se populariser. Par exemple, on sait que Nazareth avait refusé de composer des musiques pour le Théâtre de variétés, un genre musical que Chiquinha Gonzaga a aidé à consolider ${ }^{33}$.

Nazareth eut du succès comme pianiste dans les clubs des sociétés de l'élite et le salon du cinéma Odéon. Comme compositeur, il devint « le roi des tangos $»^{34}$. Afin d'éloigner son œuvre du genre populaire, il décida de nommer ses pièces musicales "tango brésilien $»^{35}$ et les jouait sur un tempo lent afin que leur aspect dansant ne ressorte. 

critiques comme compositeur érudit plutôt que comme compositeur populaire. Dans les années 1920, son originalité attira l'attention de musiciens étrangers qui contribueront à la valorisation tardive de son œuvre ${ }^{36}$. avoir reçu une solide formation au piano. Au début du vingtième siècle, le processus de spécialisation des activités artistiques s'accrut en Occident. Dans ce sillon, la figure de l'interprète ayant gagné de l'indépendance par rapport au compositeur, elle devint aussi valorisée que ce dernier. La performance musicale fut subordonnée aux qualités d'interprétation, mais au détriment de l'improvisation et de la composition ${ }^{37}$. L'ascension du piano comme instrument solo au Brésil suivit le même chemin, à savoir le développement de la spécialisation des tâches et la nécessité de formations plus précoces et individualisées pour devenir pianistes concertistes. Comme nous l'avons déjà vu, une telle formation érudite n'était pas accessible à tous, et l'histoire de Nazareth le démontre bien. quelques exemples, devinrent célèbres en interprétant leurs propres œuvres, ce n'est plus vraiment le cas au vingtième siècle, sauf quelques rares exceptions : les pianistes virtuoses sont essentiellement les interprètes des œuvres d'autres compositeurs. malgré le prix reçu et sa formation musicale au Conservatoire de Paris, avait abandonné sa carrière de pianiste, nous cherchons à présent à comprendre comment Guiomar Novaes ${ }^{38}$, Antonietta Rudge (1885-1974) ${ }^{39}$ et Magda Tagliaferro (1893-1986) ${ }^{40}$ sont devenues les premières concertistes du pays. Nous analyserons comment leur vocation ${ }^{41}$ a surgi, comment celle-ci fut reçue par la famille; nous chercherons à connaître ceux qui les ont encouragées, le chemin qu'elles ont suivi et les obstacles qui se sont imposés à elles jusqu'à ce qu'elles deviennent des professionnelles.

Ces trois pianistes commencèrent leurs carrières à Sao Paulo. Or, à la fin du dixneuvième siècle et au début du vingtième siècle, grâce au cycle du café, cette ville devint, progressivement, un important centre musical. La richesse des familles productrices, le volumineux flux d'immigrants, avec, parmi ceux-ci, des musiciens, tout cela favorisa une effervescence culturelle. C'est un groupe de fermiers qui fut à l'origine de la venue du chef d'orchestre italien Luigi Chiaffarelli (1856-1923) pour l'enseignement du piano aux jeunes filles à Sao Paulo, dès 1883. Ses compétences, son talent et son dévouement en tant que professeur contribuèrent grandement à la valorisation du piano comme instrument de concert.

41 Durant les 40 années de sa vie à Sao Paulo, il consolida une école de piano qui forma plusieurs artistes de réputation, parmi lesquels Antonietta, Guiomar et Souza Lima (1898-1982). Guiomar et Souza Lima obtinrent tous deux le prix de piano du Conservatoire de Paris. Chiaffarelli institua l'habitude de réunir ses élèves et de recevoir chez lui des musiciens étrangers de passage dans la ville. En outre, il participa à la fondation du Conservatoire Dramatique et Musical de Sao Paulo en 1906.

Antonietta fut la première élève de Chiaffarelli à obtenir une reconnaissance internationale en tant que concertiste. Enfant, elle avait déjà attiré l'attention de Chiaffarelli ainsi que celle de la presse, qui l'a toujours considérée une enfant prodige. La presse vantait ses habiletés exceptionnelles de mémorisation et de lecture ${ }^{42}$. 
43 Antonietta fut initiée au piano par sa mère, qui l'encouragea ensuite à poursuivre ces études. Elle naquit dans la capitale de Sao Paulo, en 1885, fille de João Henrique et d'Ana Emília, d'origine anglaise. Elle joua en public pour la première fois à Sao Paulo, en 1893. Plus tard, elle assura d'autres prestations publiques dans les salles de concert les plus importantes à Sao Paulo et à Rio de Janeiro ${ }^{43}$. Son talent et sa carrière, qui purent se construire grâce au soutien financier de sa famille pour la production de concerts, n'empêchèrent pas le mariage d'Antonietta, très jeune, avec son cousin d'origine anglaise, Charles Miller (1874-1953). En 1905, à l'âge de 20 ans, Antonietta partit en voyage en Europe pour la première fois, accompagnée de son mari, qui l'aida à se présenter en tant que concertiste au Bechstein Hall à Londres. Elle prolongea sa tournée en France et en Allemagne.

Cependant, après la naissance de sa fille, Antonietta n'assura que sporadiquement ses prestations de concertiste. Elle ne donna pas suite aux invitations de nouvelles tournées en Europe, car elle ne voulait pas s'absenter de son foyer. Si l'on sait que Charles Miller appréciait qu'Antonietta joue du piano, son enthousiasme face au talent de sa femme n'ira toutefois pas aussi loin que celle d'Otávio, mari de Guiomar Novaes et ancien élève de piano de Chiaffarelli, qui abandonna sa carrière d'architecte pour s'occuper personnellement de la carrière de son épouse et de sa famille. Dans les années 1920, Antonietta se sépara de Miller pour vivre avec le poète Menotti Del Picchia (1892-1988), ce qui écourta, en fait, encore plus sa carrière de concertiste. Toutefois, comme professeur de piano, elle participa de façon active à l'essor de la vie culturelle à Sao Paulo ${ }^{44}$.

Guiomar, la première élève de Chiaffarelli à recevoir le prix du Conservatoire de Paris, arriva avec sa mère à Sao Paulo grâce au soutien financier de sa marraine et amie de la famille, Alda Silva Prado. Guiomar devint l'élève du maître Chiaffarelli et, en 1902, à l'âge de huit ans, elle donna son premier concert en public.

C'est grâce à ses frères aînés, qu'elle eut son premier contact avec le piano. Née en 1894 à Sao João da Boa Vista, ville située en province de l'État de Sao Paulo, elle était la dixseptième fille des dix-neuf enfants d'Anna et de Manoel, commandant au sein des forces militaires et marchand de café. Ses sœurs aînées jouaient, elles aussi, du piano. Leur mère, frustrée dans sa quête d'une vie artistique et désirant ardemment les voir réaliser son rêve, les encouragea à parfaire leur pratique de l'instrument. Très tôt, étudier au Conservatoire de Paris figurait parmi les plans de la pianiste, encouragée en cela par sa marraine et sa mère. Peu à peu, ces études à l'étranger devinrent son plus grand rêve. En 1909, à l'âge de 15 ans, elle obtint une bourse d'études de l'État de Sao Paulo pour aller étudier en France. La même année, elle fut admise en première position au Conservatoire de Paris. En juillet 1911, ses études s'achevèrent avec l'obtention d'un premier prix de piano, ce qui fit rayonner sa réputation et facilita la commande de concerts dans les différents pays de l'Europe. La princesse Isabelle, exilée en Europe depuis la proclamation de la République en 1889, était d'ailleurs présente à l'un de ces concerts, en 1912.

Guiomar se maria en 1922. Son premier enfant naquit en 1923. Elle aura deux enfants. Après son mariage et sa maternité, elle n'abandonna pas sa carrière, car elle pouvait compter sur l'appui de son mari, qui s'occupait de la famille et de sa carrière. Grâce à lui, elle réalisa plusieurs tournées dans les salles de concert les plus importantes des États-Unis ${ }^{45}$. 

et Louise Tagliaferro, tous les deux français, passaient leurs vacances. Bien que Paulo rêvait de devenir chanteur et pianiste, son propre père l'avait empêché de devenir artiste, refusant d'avoir un fils musicien. Il préférait que son fils devienne ingénieur. Celui-ci bénéficia toutefois de leçons de chant et de piano durant ses études. Quand il arriva au Brésil, plus précisément à Sao Paulo, il abandonna finalement sa profession pour se consacrer à l'enseignement de la musique; il s'avérait en revanche trop tard pour lui de commencer une carrière de chanteur ou de pianiste ${ }^{46}$.

Ainsi, Magda grandit en écoutant les leçons données par son père. Quand elle eut cinq ans, il l'initia immédiatement au piano. À l'âge de neuf ans, elle se produisit pour la première fois en public lors d'un concert à Sao Paulo. Son père l'encouragera et l'aidera tout au long de sa carrière et ce, jusqu'à ce qu'il décède, en 1907. Il l'emmena à Paris dans le but de la préparer à son examen d'entrée au Conservatoire de la capitale française. En 1906, Magda entra au Conservatoire de Paris et, en 1907, à l'âge de 14 ans, elle obtient le premier prix du Conservatoire. Ensuite, elle effectua une tournée en France, accompagnée du compositeur français Gabriel Fauré (1845-1924), ce qui marqua le début d'une fructueuse carrière : elle reçut plusieurs prix ${ }^{47}$ et décorations ${ }^{48}$ et réalisa des tournées en Europe, aux États-Unis et au Brésil. En 1937, elle fut nommée professeur du Conservatoire de Paris par le gouvernement français, en remplacement d'Isidor Philipp (1863-1958). En raison de la Seconde Guerre Mondiale, Magda quitta le Conservatoire et Paris; concomitamment le ministre de l'éducation du gouvernement Getúlio Vargas l'invitait à revenir au Brésil comme professeur de piano. Elle s'installa donc au Brésil, enseigna, et fonda une école de piano basée sur sa propre méthode d'enseignement et sur ses propres techniques d'interprétation ${ }^{49}$.

Dans ses mémoires ${ }^{50}$, Magda évoque ses relations amoureuses qu'elle estimait des plus importantes. Sa façon de les raconter nous éclaire sur la manière dont elle menait tant sa carrière que sa vie amoureuse. Sa première relation fut avec Jules Boucherit (1877-1962), un violoniste français. Ils se fréquentèrent pendant 10 ans, chacun habitant son propre appartement. Son premier mariage fut avec André B., un administrateur rattaché à l'École Normale de Musique de Paris. Celui-ci, veuf, avait déjà deux filles, lesquelles n'aimaient pas Magda. De plus, il s'avéra très jaloux. Une fois au Brésil en raison de la guerre, elle demanda le divorce. Plus tard, elle se maria avec Victor Konn, qu'elle avait connu pendant l'une de ses nombreuses tournées en Grèce. Pendant dix ans, Victor s'occupa personnellement des biens de Magda. Cependant, en raison d'une mauvaise gestion, Magda perdit son patrimoine et se vit obligée de rembourser les dettes contractées par son mari. Elle demanda une nouvelle fois le divorce. Le retour de Magda à Paris en 1949 fut marqué par un concert qu'elle donna dans un Théâtre des Champs-Élysées bondé. Elle s'établit à Paris tout en revenant régulièrement au Brésil.

52 La trajectoire de ces pianistes résumée, il devient à présent possible de dégager quelques aspects fondamentaux permettant de comprendre l'émergence de leur carrière. Nous commencerons par l'importance de l'éducation du goût à la musique, pour lesquelles la famille joue un rôle essentiel. L'initiation précoce à l'instrument est très dépendante d'une relation de proximité avec la musique au sein de l'univers familial ${ }^{51}$.

53 Le passage " d'une pratique du piano en amateur » à une carrière professionnelle exige des encouragements et des appuis, tant de la part de la famille, que des professeurs 
expérimentés, guidant la formation, et enseignant les bonnes techniques. Ensemble, famille et professeur, assument la responsabilité de donner un élan aux premiers pas d'une profession et de la carrière.

Ce fut le cas pour Magda, dont le père exerça ces deux fonctions - celle de père et celle de professeur. Après la mort de son père, la concertiste a assumé sa vie professionnelle et affective tout en subvenant aux besoins de sa propre mère. Guiomar, elle, a su trouver en Chiaffarelli un professeur et un maître ; sa mère soutint la jeune pianiste en lui trouvant un bon professeur de piano et en lui permettant de se consacrer exclusivement à l'étude de l'instrument. Plus tard, c'est Otávio qui prit la relève et occupa cette place pour favoriser la carrière et la vie personnelle de Guiomar.

L'étude de la trajectoire d'Antonietta a mis en évidence les principales raisons qui ont provoqué l'interruption prématurée de sa carrière. L'appui de ses parents au début de sa carrière ne fut pas suffisant pour lui permettre de renoncer à une destinée convenue, liée à une position élevée dans la hiérarchie sociale. Son éducation lui a fait connaître le même sort que celui des autres jeunes filles raffinées : se marier tôt pour assurer et maintenir le statut social de la famille. De plus, selon ce qu'affirme sa fille Helena, sa mère n'avait jamais vraiment eu l'intention de se construire une carrière professionnelle; on pouvait constater chez Antonietta un certain manque d'ambition professionnelle. Aussi, il semble qu'elle n'aimait pas jouer devant un public ${ }^{52}$.

Bien que le mariage et la maternité représentaient les obstacles principaux et les plus communs pour une carrière, Magda et Guiomar ont réussi à transgresser les conventions du genre. Leur réussite s'explique, d'une part, par l'appui inconditionnel de leur famille, qui n'a pas hésité à les dispenser des tâches maternelles et conjugales en faveur de leur carrière, et d'autre part, par l'indépendance que leur accordait leur carrière internationale pour mener leur vie de la façon dont elles l'entendaient. Dans la mesure où elles séjournaient longtemps à l'étranger, elles étaient moins soumises aux préjugés et aux jugements qui prévalaient alors dans la société brésilienne. Malgré ces redoutables obstacles, ces femmes évoluèrent dans des conditions leur permettant de parfaire une technique particulière très spécialisée, celle du piano et ce, de façon précoce.

Il faut souligner que la préparation de l'artiste implique l'acquisition de dispositions corporelles et mentales permettant l'apprivoisement du risque intrinsèque de jouer devant le public. Un concert exige une performance publique de l'artiste, une situation difficile pour les femmes qui n'avaient peu ou jamais l'opportunité de s'exposer et pratiquer devant un public au-delà du salon de leur foyer. Malgré une formation depuis l'enfance pour jouer en public, les femmes ne réussissaient pas toujours à se sentir " socialement à l'aise ". Il leur était difficile d'assumer toutes les exigences liées à une carrière artistique. Antonietta Rudge en est un exemple : malgré son aisance technique, sa créativité et son talent, elle n'aimait pas se produire devant un public. Peut-être est-ce pour cette raison qu'elle a finalement décidé de ne pas prioriser sa carrière ?

Bien que les carrières de compositeur et d'interprète se soient progressivement séparées, cela ne signifiait pas qu'il était impossible pour les musiciens de passer d'une profession musicale à une autre au cours de leur vie. Il est important souligner qu'à cette époque, les femmes n'avaient pas un libre accès à tous les métiers musicaux. Par exemple, la composition et la direction d'un orchestre étaient des "vocations" majoritairement pratiquées par des hommes. En ce sens, l'analyse de la carrière de Souza Lima (1898-1982) démontre bien ce phénomène : il a commencé sa carrière 
comme pianiste-interprète et compositeur, et à la fin de sa vie, il devint chef d'orchestre.

Souza Lima ${ }^{53}$, né en 1898 à Sao Paulo, fut le deuxième élève de Chiaffarelli à obtenir le prix du Conservatoire de Paris. Il devint l'élève du prestigieux professeur en 1910. De la même façon que les femmes pianistes vues précédemment, la famille de Souza Lima offrait un environnement propice à l'initiation musicale; d'ailleurs tous ses frères et sœurs apprirent à jouer du piano. Sa mère l'a toujours encouragé et son frère aîné, Paulo Augusto, fut un exemple de professionnalisme. Selon Lima, Paulo Augusto fut l'un des premiers pianistes masculins à se consacrer à l'étude du piano dans le but de devenir concertiste ${ }^{54}$.

Il semble que Souza Lima fut, tout comme son frère, parmi les rares hommes de son époque à avoir pu devenir pianiste-interprète. Selon ce qu'il rapporta, de tous les élèves de Chiaffarelli, il était le seul homme. C'est sous l'influence et la direction de son frère que Souza Lima, à l'âge de quatre ans, fit ses premiers pas en musique. Mais il ne devint l'élève du professeur Chiaffarelli qu'à l'âge de 12 ans, son frère ayant été surchargé par les impératifs liés à sa propre carrière. C'est Chiaffarelli lui-même qui recommanda au jeune Souza Lima d'étudier la composition auprès du professeur Agostinho Cantú - sans abandonner l'apprentissage du piano. Peu après, le jeune apprenti de composition prit le risque de participer à deux concours de composition ${ }^{55}$.

61 En 1919, alors âgé de 21 ans, après avoir reçu une bourse d'études du Pensionnat Artistique de l'État de Sao Paulo, Souza Lima entra au Conservatoire de Paris ${ }^{56}$. Il obtint le premier prix de piano en 1922, à l'âge de 24 ans. Ainsi, entrait-il au Conservatoire bien plus âgé que les femmes pianistes que nous avons étudiées. En fait, en raison de la première Guerre mondiale, les âges d'admission au Conservatoire avaient été élargis, et Souza put bénéficier de cette opportunité. Sinon, il n'aurait probablement jamais pu étudier à Paris. Dans son autobiographie, en revenant sur son entrée au Conservatoire, il mit en évidence l'importance de cette école et de son prix qui permirent à sa carrière de prendre son envol.

62 Assurément, ce diplôme et ce prix furent les jalons symboliques nécessaires à Souza Lima pour qu'il puisse se définir comme " un vrai artiste », malgré ses longues années d'étude et de prestige qui lui valaient déjà une solide réputation de pianiste et compositeur dans les milieux artistiques et intellectuels de la ville.

En 1930, Souza Lima revint dans son pays, se maria et repartit dans la capitale française avec son épouse. Il effectua des tournées internationales et, plus tard, au Brésil. Au Brésil, il devint chef d'orchestre de l'Orchestre municipal de Sao Paulo, position qu'il occupera et à laquelle il se vouera durant 32 ans.

Dans cet article, nous avons tenté de dégager, en mettant en perspective divers profils, les circonstances et les conditions sociales essentielles à la construction d'une carrière musicale parmi les pianistes, en montrant en quoi la pratique du piano était distincte entre hommes et femmes et au travers des différentes classes sociales.

La carrière de concertiste n'est devenue un choix envisageable au Brésil qu'au début du vingtième siècle avec la consolidation de l'enseignement du piano érudit, et l'arrivée de plusieurs pianistes étrangers qui contribuèrent à assoir la renommée du récital. Cette carrière, extrêmement spécialisée, s'est développée au sein d'un processus de formalisation et de hiérarchisation de la structure de l'univers artistique. 

un univers masculin n'était pas une option pour les jeunes filles d'un rang social élevé. De plus, la musique populaire ne faisait alors pas partie du répertoire transmis par les professeurs privés de piano, et surtout pas par les professeurs étrangers.

71 Malgré ces restrictions de genre et de classe sociale, les femmes furent encouragées à jouer du piano dès le dix-neuvième siècle. Au début du vingtième siècle, c'est justement ces conditions qui permirent à des femmes de devenir les premières personnes à être consacrées concertistes au Brésil. Les circonstances qui, jadis, leur portaient préjudice, les ont motivées à développer une carrière de pianiste-interprètes, beaucoup plus que les hommes.

72 Pour les hommes, jouer du piano n'était «qu'un choix difficile $»^{58}$, parmi un large éventail d'activités musicales qu'ils pouvaient librement adopter. Deux situations ont été relevées : dans un cas, le devoir de subvenir à leurs besoins et à ceux de leur famille leur imposait de trouver rapidement du travail: ils enseignaient, jouaient et composaient des mélodies de différents styles pour différents milieux, mais cela se fit au détriment d'une véritable spécialisation. Dans un autre cas, leur vocation artistique fut entravée par leurs parents, mésestimant les professions musicales et orientant leur 
fils vers des métiers plus appréciés, générant des revenus et une élévation dans la hiérarchie sociale. Ce point de vue était commun à l'époque, comme nous l'avons vu dans l'histoire de Nazareth et Paulo Tagliafero, le père de Magda.

Ainsi, on peut affirmer que le développement et la consolidation de la carrière de concertiste dépendaient de plusieurs facteurs : l'encouragement et la reconnaissance par la famille d'une vocation musicale de manière précoce, l'astreinte aux études pour une formation érudite rigoureuse, les prix et les réussites lors de concours dans les conservatoires européens et les institutions internationales.

Pour conclure, nous ne pouvons pas dire que jouer du piano au dix-neuvième siècle ne concernait que les jeunes filles de classes sociales privilégiées et constituait une pratique de salon «banalisée ", qui, au vingtième siècle et pour quelques rares femmes, se serait transformée en une pratique professionnelle facilitant une riche carrière. La réalité est plus complexe. Notre propos démontre que son exercice pouvait être à la fois féminin, masculin, populaire, érudit, amateur, professionnel, etc. Autrement dit, il n'existait pas qu'un seul type de pratique, mais bien plusieurs, et, pour les comprendre, il est nécessaire de considérer les clivages de genre et de classes sociales.

La carrière de pianiste-interprète devint possible grâce à une certaine ouverture provoquée par la vie cosmopolitaine qui s'affirma au début du vingtième siècle à Rio de Janeiro et à Sao Paulo, favorisant du même coup la vocation et l'ambition d'une génération de femmes, qui ont éprouvé la nécessité de faire évoluer les normes sociales. Les écoles de musique, comme l'Institut National de Musique, à Rio de Janeiro, et le Conservatoire Dramatique et Musical à Sao Paulo, sont devenus des espaces intermédiaires d'apprentissage et d'études, entre la maison et la scène, où ces femmes purent vivre une nouvelle forme d'éducation et de sociabilité, tout en ouvrant leurs réseaux de relations pour la construction de leur carrière professionnelle. Ainsi, plusieurs femmes ont donné une nouvelle direction au rôle social dans lequel elles avaient été élevées.

$76 \mathrm{Si}$, au dix-neuvième siècle, les hommes et les femmes jouant du piano évoluaient dans des mondes distincts, au vingtième siècle, le piano a constitué un pont qui a permis aux femmes d'accéder à des carrières traditionnellement "masculines" dans l'univers musical. Lorsque la carrière d'un pianiste connaissait un moment un moindre succès, cette difficulté n'engendrait pas nécessairement l'interruption définitive de sa carrière professionnelle. Le riche bagage musical obtenu à travers l'étude du piano a donné aux musiciennes une assise les encourageant à prendre de nouveaux risques, et fut un levier leur permettant de se tourner vers d'autres positions dans l'univers musical professionnel, lorsqu'elles ne voulaient plus poursuivre une carrière de concertistes. Ainsi, investirent-elles de nouveaux terrains, tels que la composition, la direction d'orchestre et la musicologie, des champs qui étaient traditionnellement occupés par les hommes.

Dans l'ouvrage que nous avons publié ${ }^{59}$, nous avons étudié la trajectoire de deux autres musiciennes, Helza Camêu (1903-1995) et Joanídia Sodré (1903-1975), en analysant les croisements entre genre, corps et classe sociale dans l'univers de la musique érudite au Brésil de la première moitié du vingtième siècle. Ces deux musiciennes, bien qu'elles aient commencé leur carrière musicale comme pianistes dans les années 1920 à Rio de Janeiro, ne poursuivirent pas une carrière de concertiste. choisi de se hisser à des positions traditionnellement masculines : Helza fut couronnée 
de multiples prix en tant que compositrice et musicologue (elle s'intéressait à la musique autochtone), alors que Joanídia devint chef d'orchestre en 1930 et fut la première femme à diriger l'Institut National de Musique ${ }^{60}$.

\section{BIBLIOGRAPHIE}

AULER, Guilherme. (1956). Os bolsistas do Imperador advogados, agrônomos, arquiteto, aviador, educação primária e secundária, engenheiros, farmacêuticos, médicos, militares, músicos, padres, pintores, professores. Petrópolis: Tribuna de Petrópolis.

AZEVEDO, Luis Heitor Corrêa de. (1956). 150 anos de música no Brasil (1800-1950). Rio de Janeiro: José Olympio.

BARONCELLI, Nilcéia. Cleide da Silva. (1987). Mulheres compositoras. São Paulo: Roswith Kempf Editores.

BARONI, Silvio Ricardo. (1999). O intérprete-pianista no fim do milênio. Thèse de Doctorat,Département de Musique del'Université de Sao Paulo (USP).

BOCCANERA JÚNIOR, Sílio. [1903,1904] (1988). Perfil Biográfico de Luiza Leonardo. In : Revista do Grêmio Literário (edição fac-similar). Salvador: Artes Gráficas, p. 414-417, p. 438-444.

BOURDIEU, Pierre. (1976). Anatomie du Goût. Actes de la recherche en sciences sociales. vol. 2, $\mathrm{n}^{\circ} 5$, p. $18-43$.

CACCIATORE, Olga Gudolle. (2005). Dicionário Biográfico de Música Erudita Brasileira. Rio de Janeiro: Forense Universitária.

CARVALHO, Dalila Vasconcellos de. (2012). o gênero da música. A construção social da vocação. São Paulo: Editora Alameda / Fapesp.

CARVALHO, Vânia Carneiro de. (2008). Gênero e Artefato: O sistema doméstico na perspectiva da cultura material - São Paulo, 1870-1920. São Paulo: Edusp/Fapesp.

DINIZ, André. (2008). Joaquim Callado : O pai do choro. Rio de Janeiro: Jorge Zahar Editor.

DINIZ, Edinha. (2005). Chiquinha Gonzaga : uma história de vida. Rio de Janeiro: Ed. Rosa dos Tempos.

ESCAL, Françoise (1999). Approche Globale. Hypothèse Culturaliste. Hypothèse Naturaliste. In : Rousseau-Dujardin, Jacqueline. \& Françoise, Escal. Musique et Diférence des Sexes. Paris :

L'Harmattan. p. 19-110.

GUÉRIOS, Paulo Renato. (2003). Heitor Villa-Lobos. Rio de Janeiro: Editora FGV.

LEITE, Edson. (2001). Magdalena Tagliaferro : testemunha de seu tempo. São Paulo: Annablume.

LÉPRONT, Catherine. (1990). Clara Schumann. São Paulo: Editora Martins Fontes.

LIMA, João de Souza. (1982). Moto perpetuo : a visão poética da vida através da música. São Paulo: IBRASA. 
MACHADO, Cacá. (2007). O Enigma do homem célebre : Ambição e vocação de Ernesto Nazareth. São Paulo: Instituto Moreira Salles.

MARCONDES, Marcos Antônio. (Ed.). (2000). Enciclopédia da Música Brasileira : popular, erudita e folclórica. São Paulo: Art Editora et Publifolha.

MARIZ, Vasco. (2002). A canção brasileira de câmara. Rio de Janeiro: Francisco Alves.

MARIZ, Vasco. (2005). História da Música no Brasil. Rio de Janeiro: Editora Nova Fronteira.

NEEDELL, Jeffrey D. (1993). Belle Époque Tropical: Sociedade e cultura de elite no Rio de Janeiro na virada do século. São Paulo: Companhia das Letras.

ORSINI, Maria Stella. (1992). Guiomar Novaes: uma arrebatadora história de amor. São Paulo: Editora C.I.

PAOLA, Andrely e BUENO, Helenita (1998). Escola de Música da Universidade Federal do Rio de Janeiro : História e Arquitetura. Rio de Janeiro: UFRJ.

PEREIRA, Avelino Romero. (2007). Música, Sociedade e Política: Alberto Nepomuceno e a República Musical. Rio de Janeiro: Editora UFRJ.

RAVET, Hyacinthe. (2005). Féminin et Masculin en musique. Dynamiques identitaires et rapports de pouvoir. In : Green, Anne-Marie et Ravet, Hyacinthe. L'accès des femmes à l'expression musicale. Apprentissage, création, interprétation : les musiciennes dans la société. Paris : L'Harmattan.p. 225-246.

RAVET, Hyacinthe. (2007). Devenir clarinettiste : Carrières féminines en milieu masculin. In : Actes de la Recherche en Sciences Sociales, Paris, nº 168, p. 51-67.

REZENDE, Carlos Penteado. (1970). Notas para uma história do piano no Brasil (século XIX). In : Revista Brasileira de Cultura, Rio de Janeiro, n. 6, p. 09-38.

SAID, Edward. (1991). A performance como situação extrema. In : Elaborações Musicais, Rio de Janeiro : Imago, p. 27-71.

SAPIRO, Gisèle. (2007). La vocation artistique entre don et don de soi. In : Actes de la Recherche en Sciences Sociales, Paris, $\mathrm{n}^{\circ}$ 168, p. 5-12.

SEVCENKO, Nicolau. (1985). Literatura como missão : tensões sociais e criação cultural na Primeira República. São Paulo: Editora Brasiliense.

SHLOCHAUER, Regina. (1992). A presença do piano na vida carioca no século passado. Mémoire de maîtrise, Département de Musique de l'Université de Sao Paulo (USP).

SILVA, Eliana Maria de Almeida. (2008). Clara Schumann : Compositora X Mulher de compositor. Mémoire de maîtrise, Département de Musique de l'Université de Sao Paulo (USP).

SIMIONI, Ana Paula Cavalcanti. (2008). Profissão Artista : pintoras e escultoras acadêmicas brasileiras. São Paulo : Edusp.

TAGLIAFERRO, Magdalena (1979). Quase Tudo... (Memórias). Rio de Janeiro: Editora Nova Fronteira.

TOFFANO, Jaci. (2007). As pianistas dos anos 1920 e a geração jet-lag : o paradoxo feminista. Brasília: Editora UNB.

WISNIK, José Miguel. (1977). o Coro dos contrários : a música em torno da semana de 22. São Paulo: Duas Cidades. 


\section{NOTES}

2. MACHADO, Cacá. O Enigma do homem célebre: Ambição e vocação de Ernesto Nazareth, p. 27.

3. Aujourd'hui, Conservatoire National Supérieur de Musique et Danse de Paris.

4. L'événement symbolique principal marquant l'entrée dans cette période est l'ouverture de l'Avenida Central (aujourd'hui Av. Rio Branco), un grand boulevard inauguré en 1904. Parmi les principaux édifices publics qu'on érigea sur cette avenue, on compte : le Teatro Municipal (1909), le Palais Monroe (1906), la Bibliothèque Nationale (1910) et l'École Nationale de Beaux-Arts (1908). In : NEEDELL, Jeffrey D. Belle Époque Tropical: Sociedade e cultura de elite no Rio de Janeiro na virada do século, p. 61.

5. C'est-à-dire : réprobation contre les habitudes et les mœurs liées à la société traditionnelle «la négation de tout élément » de la culture populaire; la nouvelle urbanisation de la ville, qui avait expulsé la population pauvre du centre. Celui-ci fut isolé pour que les élites puissent en jouir. In :SEVCENKO, Nicolau. Literatura como missão: tensões sociais e criação cultural na Primeira República, p. 30.

6. Carlos Gomes (1836-1896) écrit de nombreuses «modinhas» qui sont devenues populaires. Quelques-unes furent écrites en italien ou en français. Elles ne figuraient pas dans le répertoire érudit. In :MARIZ, Vasco. A canção brasileira de câmara.

7. En 1911, le compositeur Alberto Nepomuceno ne voulait pas voir son nom être lié aux opérettes, alors perçues par les compositeurs érudits comme étant un genre musical inférieur. Ainsi, il signa un contrat selon lequel ses œuvres seraient publiées sous le nom de João Valdez et la maison d'édition ne devait pas divulguer le nom du compositeur. In :PEREIRA, Avelino Romero. Música, Sociedade e Política : Alberto Nepomuceno e a República Musical, p. 225.

8. Le Théâtre de variétés (ou la Revue Théâtrale ) arriva au Brésil en 1859. Il s'agissait d'une pièce musicale comique ou satirique sur des sujets d'actualité. Elle s'inspirait du vaudeville français. In : Diniz, Edinha. Chiquinha Gonzaga : uma história de vida, p. 115.

9. Bien que quelques sources historiques indiquent la présence du piano depuis la période coloniale, c'est suite à l'arrivée de la cour portugaise en 1808 à Rio de Janeiro que le « cycle du piano » commence. L'ouverture des ports et les traités signés entre le Portugal et l'Angleterre permirent une arrivée massive de pianos au Brésil. D'un autre côté, Rio de Janeiro devint le centre culturel et intellectuel du pays, notamment de l'univers musical. In : REZENDE, Carlos Penteado. Notas para uma história do piano no Brasil (século XIX), p. 14.

10. Il s'agit du nom péjoratif donné aux musiciens qui avaient peu de formation musicale et beaucoup de rythme. In :MACHADO, Cacá. O Enigma do homem célebre : Ambição e vocação de Ernesto Nazareth, p. 20.

11. PEREIRA, Avelino Romero. Música, Sociedade e Política : Alberto Nepomuceno e a República Musical, p. 226.

12. GUÉRIOS, Paulo Renato. Op. cit. p. 88.

13. Le terme "musique occidentale » se réfère à une expression esthétique globale revendiquée par une élite musicale, provenant d'Italie, d'Allemagne et de France, qui « a la primauté dans la définition des formes valables de l'art, ou selon leurs propres termes, dans la définition des formes universelles... » de l'expression musicale. C'est selon les paramètres établis par les « centres de la musique occidentale » que les musiciens et la production musicale des "autres pays » devaient construire leurs manifestations musicales, afin d'être considérées légitimes. In : GUÉRIOS, Paulo Renato. Heitor Villa-Lobos, p. 82.

14. Le professeur de chant, Arcângelo Fiorito (1813-1887), enseignait le piano une fois par semaine aux personnes intéressées. En 1873, le pianiste et compositeur Carlos Cavalier Darbilly (1846-1918) s'est proposé pour donner des leçons de piano gratuitement au Conservatoire Impérial pendant dix ans. In:Shlochauer, Regina. A presença do piano na vida carioca no século passado,1992, p. 110. 
15. Arthur Napoleão était propriétaire de la maison d'édition Casa Editorial Arthur Napoleão \& Miguez.

16. À cette époque, il y avait deux choix pour publier une partition musicale : ou le compositeur vendait l'œuvre à l'avance, ou encore il assumait les frais de l'édition en échange des bénéfices de la vente. In :PEREIRA, Avelino Romero. Música, Sociedade e Política: Alberto Nepomuceno e a República Musical, p. 25.

17. En tout, 151 boursiers bénéficièrent de ces bourses, parmi lesquels seulement 12 étaient des musiciens. De plus, on n'y compte que deux femmes : la cantatrice Maria Monteiro (1870-1897), née à Campinas, qui a reçu l'aide financière pour étudier à Milan en 1887 , et la pianiste Luiz Leonardo, en 1873. In : AULER, Guilherme.

18. BOCCANERA JÚNIOR, Sílio. Perfil Biográfico de Luiza Leonardo.

19. Plus tard appelé Institut Benjamin Constant, l'institut fut fondé pour offrir aux malvoyants des cours les préparant à exercer un art, un artisanat ou une profession libérale. Parmi d'autres, retenons : organiste, accordeur de piano, professeur de musique.

20. L'orchestre fut créé en 1861 par le compositeur Jules Pasdeloup (1819-1887) dû aux Concerts Populaires. Le but de ces concerts était d'augmenter le public des concerts et la connaissance de nouveaux compositeurs et musiciens qui n'étaient pas exclusivement français. Il était formé de 80 musiciens et présentait des concerts donnés le dimanche au Cirque d'Hiver. Ses activités furent interrompues en 1886 et reprirent en 1919. Encore actif aujourd'hui, il s'agit du plus ancien l'orchestre symphonique de France.

21. TOFFANO, Jaci. As pianistas dos anos 1920 e a geração jet-lag. O paradoxo feminista, p. 63.

22. BARONI, Silvio Ricardo. O intérprete pianista no fim do milênio, p. 55, 62.

23. Les clubs musicaux étaient des associations privées de musique dont les membres provenaient de la cour et de son élite environnante. Ils avaient été constitués pour encourager l'essor du concert de chambre et du concert symphonique ainsi que pour propager le répertoire moderne (par exemple, Richard Wagner, Camille Saint-Saëns) au détriment de l'opéra. In :AZEVEDO, Luis Heitor Corrêa de. 150 anos de música no Brasil (1800-1950).

24. TOFFANO, Jaci. As pianistas dos anos 1920 e a geração jet-lag. O paradoxo feminista, p. 64 .

25. Par exemple, dans l'un des clubs les plus importants, le Club Beethoven, établi en 1882 et dont le Comte d'Eu (1842-1922) devint président honoraire, l'accès aux femmes fut interdit jusqu'en 1888. In : Op. cit. p. 62.

26. Nous utilisons ici le terme professeure, dans sa forme féminisée, pour souligner qu'il s'agit de la première femme enseignant au Conservatoire.

27. PAOLA, Andrely ; BUENO, Helenita (1998). Escola de Música da Universidade Federal do Rio de Janeiro : História e Arquitetura, p. 28.

28. Il est important souligner que certains instruments musicaux étaient plus appropriés pour les femmes. Par exemple, le piano était un instrument qui convenait aux jeunes filles plus qu'aucun autre instrument parce que (...) «elles pouvaient jouer du piano assises, les jambes fermées, sans faire de grands mouvements - en plus, elles ne restaient pas devant le public en faisant des grimaces ou en mouvant le corps ». In:SILVA, Eliana Maria de Almeida. Clara Schumann: Compositora X Mulher de compositor, p. 74.

29. Le même constat s'applique au sujet du Conservatoire de Paris, établi en 1795 : Cette institution n'excluait pas les femmes, mais était encadrée par un ensemble de règles qui séparaient les hommes et les femmes. En effet, on y établissait des règles différentes pour chacun des groupes, auxquels on attribuait des classes et des professeurs distincts. En outre, les professeurs enseignant aux groupes de femmes étaient moins payés que ceux enseignant aux groupes d'hommes. ESCAL, Françoise. Approche Globale. Hypothèse Culturaliste. Hypothèse Naturaliste. In : Rousseau-Dujardin, Jacqueline. \& Françoise, Escal. Musique et Différence des Sexes, p. 79, 83.

30. Diniz,Edinha. Chiquinha Gonzaga : uma história de vida. 
31. CARVALHO, Vânia Carneiro de. Gênero e Artefato : O sistema doméstico na perspectiva da cultura material-São Paulo, 1870-1920, p. 156, 157.

32. Joaquim Antônio Callado créa un groupe musical nommé Choro Carioca. Ce fut là la première référence au terme «choro ». Ce groupe était constitué d'une flûte traversière pour la mélodie, de deux violons et du cavaquinho(petite guitare à quatre cordes). Le Choro est né de l'improvisation.In: Diniz, Edinha.Chiquinha Gonzaga : uma história de vida, p. 93.

33. MACHADO, Cacá. O Enigma do homem célebre: Ambição e vocação de Ernesto Nazareth, p. 97. 34. MACHADO, Cacá. Op. cit., p. 90, 97.

35. Le tango brésilien est une version stylisée du maxixe. Il n'a aucun rapport avec le tango argentin.

36. Dans un article paru en 1920, le compositeur français Darius Milhaud (1892-1974) décrit Nazareth comme un génie. In : Wisnik, José Miguel.o coro dos contrários : a música em torno da semana de 22, p. 39.

37. SAID, Edward. A performance como situação extrema.

38. Les données sur Guiomar Novaes ont été obtenues chez ORSINI (1992).

39. Les données sur Antonietta Rudge ont été obtenues chez Toffano (2007), Marcondes (2000).

40. Les données sur Magdalena Tagliaferro ont été obtenues chez TAGLIAFERRO (1979), LEITE (2001).

41. La vocation comme fait social: «Malgré leur caractère subjectif et leur indétermination apparente, il existe bien des conditions sociales de formation et de matérialisation des vocations artistiques ». In: Sapiro, Gisèle. Actes de la Recherche en Sciences Sociales, Paris, no 168, p. 9.

42. Avant l'arrivée de l'enregistrement musical, la presse écrite spécialisée, surtout les journaux, contribuait à immortaliser le moment fugace des performances en concert. La presse louait les particularités de chaque artiste; elle contribua à la consolidation de leurs "noms propres » et leur image de "virtuoses" ou d'« enfants prodiges » comme si ceux-ci avaient une maitrise technique, ce qui éloigne l'artiste de l'auditeur, le professionnel de l'amateur. In : TOFFANO, Jaci. As pianistas dos anos 1920 e a geração jet-lag. O paradoxo feminista, p. 35,36.

43. Antonietta s'est produite au Clube Internacional, au Clube Germânica, au Salon Steinway, au Théâtre Municipal de Sao Paulo et à l'Institut National de Musique à Rio de Janeiro.In :TOFFANO, Jaci. As pianistas dos anos 1920 e a geração jet-lag. O paradoxo feminista, p. 100.

44. En 1912, elle participa à la fondation de la Société de Culture Artistique à Sao Paulo qui est encore active aujourd'hui. In:TOFFANO, Jaci. Op. cit. p. 100.

45. Guiomar reçut en 1939 l'ordre national de la Légion d'honneur de France et la Ordem do Cruzeiro do Sul en 1956, une décoration honorifique brésilienne. In: Cacciatore, Olga. Dicionário Biográfico de Música Brasileira, p. 318.

46. TAGLIAFERRO, Magdalena. Quase tudo... (Memórias), p. 9.

47. Parmi les prix que Magda reçut, soulignons le Grand Prix du Disque en France qu'elle reçut trois fois, en 1931, 1934 et 1981. Elle mérita ce dernier pour son disque d'interprétations d'œuvres à quatre mains et de duos pour deux pianos du compositeur français Gabriel Fauré (1845-1924). In: Leite, Édson. Magdalena Tagliaferro : Testemunha de seu tempo, p. 77.

48. Magda reçut en 1953 l'insigne de commandeur de l'ordre national de la Légion d'honneur en France et la Ordem de Rio Branco en 1972, une décoration honorifique brésilienne. In: Leite, Édson, op. cit. p. 145.

49. La Fondation Magda Tagliaferro fut créée en 1969 et son objectif est d'assurer la pérennité de l'héritage de l'artiste. Pour savoir plus, http://www.magdatagliaferro.com.br/pianista/.

50. TAGLIAFERRO, Magdalena. Quase tudo... (Memórias).

51. «... la musique, ce ne sont pas les disques et l'électrophone de ses vingt ans, grâce auquel on a découvert Bach et Vivaldi, mais le piano de famille, entendu dès l'enfance et vaguement pratiqué jusqu'adolescence; la peinture, ce ne sont pas les musées, soudain découverts dans le 
prolongement d'un apprentissage scolaire, mais le décor de l'univers familier.» In: Bourdieu, Pierre. Actes de la recherche en sciences sociales. vol. 2, $\mathrm{n}^{\circ} 5, \mathrm{p} .28$.

52. RUDGE apud TOFFANO, Jaci. As pianistas dos anos 1920 e a geração jet-lag. O paradoxo feminista, p. 102, 103.

53. LIMA, João de Souza. Moto perpetuo : a visão poética da vida através da música.

54. LIMA, João Souza, op. cit. p. 18.

55. Le premier fut un concours de composition, dans le cadre duquel Souza Lima reçut le premier prix avec le tango Então, té logo, et, il obtint le deuxième prix dans le second concours, destiné à la musique symphonique. In: LIMA, João de Souza. op. cit.

56. Le Pensionnat Artistique est une institution qui fut créée en 1912 et fermée en 1931 par le gouvernement de l'Etat de Sao Paulo afin de promouvoir la production artistique en attribuant des bourses d'études permettant aux artistes que la commission considérait méritoires d'aller étudier en Europe. Dans cette période, l'État n'avait aucune faculté ou cours supérieur d'art.

57. TOFFANO, Jaci. As pianistas dos anos 1920 e a geração jet-lag. O paradoxo feminista, p. 78, 79 et 85 .

58. « ...les représentations et images sociales associées aux instruments renvoient à des attitudes corporelles normées et contribuent largement à définir les "choix» (ou les non-choix) des instruments de musique par des filles et des garçons, choix indirectement ou directement influencés par l'entourage familial et la communauté d'éducation à la musique (que le cadre soit celui d'un conservatoire, d'une école de musique, d'une fanfare ou d'un orchestre d'harmonie, par exemple.). » In : Ravet, Hyacinthe. Féminin et Masculin en musique. Dynamiques identitaires et rapports de pouvoir, p. 231.

59. In:Carvalho, Dalila Vasconcellos de. 0 gênero da música. A construção social da vocação.

60. Aujourd'hui, l'École de Musique de l'Université Fédérale de Rio de Janeiro.

\section{RÉSUMÉS}

Dans cet article, nous analysons comment Guiomar Novaes (1894-1979), Antonietta Rudge (1885-1974) et Magda Tagliaferro (1893-1986) sont devenues les premières concertistes du Brésil et les raisons pour lesquelles des femmes, plutôt que des hommes, furent les premières à atteindre ce statut dans un contexte marqué par deux normes sociales de la fin du dix-neuvième siècle qui rendaient presque impossible une telle carrière : les restrictions de genre, confinant les femmes à pratiquer le piano de façon dilettante, et l'émergence tardive de récitals de piano. Nous pouvons mieux comprendre leur ascension en considérant de façon parallèle les trajectoires d'autres pianistes comme Ernesto Nazareth (1863-1934), Luiza Leonardo (1859-1934), Chiquinha Gonzaga (1847-1935) et Souza Lima (1898-1982).

In this paper, we analyze how Guiomar Novaes (1894-1979), Antonietta Rudge (1885-1974) and Magda Tagliaferro (1893-1986) became the first Brazilian soloists and the reasons why women, rather than men, were the first to achieve this status in a context marked by two social norms of the late nineteenth century. The gender restrictions, confining women to practice the piano as amateurs, and the fact the solo piano concerts were in their beginning in Brazil made it almost impossible the development of a career. We can better understand their ascent by considering parallel paths other pianists as Ernesto Nazareth (1863-1934), Luiza Leonardo (1859-1934), Chiquinha Gonzaga (1847-1935) and Souza Lima (1898-1982). 
INDEX

Mots-clés : pianistes brésiliennes, musique et genre, vocation, anthropologie de la musique classique occidentale

Keywords : brazilian pianists, music and gender, vocation, anthropology of Western classical music

\section{AUTEUR}

DALILA VASCONCELLOS DE CARVALHO

Maîtrise en anthropologie sociale à l'Université de Sao Paulo (USP) en 2011. 